\title{
DISEÑO, CONSTRUCCIÓN, CARACTERIZACIÓN Y ENSAYO DE PRUEBA DE UN TÚNEL DE VIENTO VERTICAL, PARA ENSAYOS DE CAIIDA LIBRE DE CUERPOS
}

\author{
Santiago Garro ${ }^{1, *}$, Santiago Pezzotti ${ }^{2,+}$, Vicente Nadal Mora ${ }^{3, \ddagger}$ \\ Desarrollo, Extensión y Transferencia - Grupo de Transporte Aéreo - Grupo de Ingeniería \\ Aplicada a la Industria), Dto. Aeronáutica, Facultad de Ingeniería, Universidad Nacional de La \\ Plata, 1900 Buenos Aires, Argentina.
}

Recibido: 30/septiembre/2019

Aceptado: 14 /octubre/2019

DOI: https://doi.org/10.5377/ce.v12i2.10344

\begin{abstract}
RESUMEN
En el presente trabajo se detalla el proceso de diseño, construcción y caracterización de un túnel de viento vertical para el ensayo de objetos en caída libre. El túnel de viento constituye un equipo especial para pruebas experimentales, en el que se busca conocer la interacción entre un cuerpo en estudio y el flujo uniforme de aire a diversas condiciones de velocidad, con la finalidad de evaluar el comportamiento fluidodinámico, energético, aerodinámico y estabilidad del cuerpo en estudio, con la particularidad de que el flujo de aire circula de abajo hacia arriba, simulando una condición de vuelo casi libre del cuerpo. El túnel de viento está compuesto por una cámara o sección de pruebas, un ventilador encargado de generar la corriente de aire, conductos y adaptadores, y la instrumentación necesaria para la realización de mediciones. El presente túnel fue desarrollado principalmente con el objeto de ensayar pararrotores. Estas sondas poseen como objeto principal frenar la caída libre de un cuerpo en la atmósfera. Existen múltiples aplicaciones de estos deceleradores, como la recuperación de elementos provenientes del espacio, la estabilización del giro de proyectiles, el transporte de cargas con precisión y el uso de sondas meteorológicas. Así mismo, tienen una potencial aplicación en el envío de sensores donde el riesgo de ingreso es muy elevado, como ser incendios o zonas afectadas por derrames tóxicos o bacteriológicos.
\end{abstract}

Palabras clave: Túnel de viento, caída libre, pararrotores.

*santiago.garro87@gmail.com

† santiago.pezzotti@ing.unlp.edu.ar

‡vnadal@ing.unlp.edu.ar 


\section{ABSTRACT}

In the present work the process of design, construction and characterization of a vertical wind tunnel for the test of objects in free fall, in particular aerodynamic rotary wing decelerators (pararotors) is detailed. The wind tunnel is a special equipment for experimental tests, which seeks to know the interaction between a solid body under study and the uniform flow of air at various speed conditions, in order to evaluate the fluid dynamic behavior, energy, aerodynamic and stability of the body under study, with the particularity that the air flow circulates from bottom to top, simulating an almost free flight condition of the body. Mainly the wind tunnel consists of a camera or test section, a fan responsible for generating the air flow, a special geometric structure according to the needs, ducts and adapters, and the necessary instrumentation for the measurements. The present tunnel was developed mainly for the purpose of testing pararotors. These probes have as main object to stop the free fall of a body in the atmosphere. There are multiple applications of these decelerators, such as the recovery of elements from space, the stabilization of the rotation of projectiles, the transport of loads with precision and the use of meteorological probes. Likewise, they have a potential application in sending probes where the risk of entry is very high, such as fires or areas affected by toxic or bacteriological spills.

Keywords: Wind tunnel, free fall, pararotors.

\section{Introducción}

Los pararrotores conforman una de las principales líneas de investigación de la UIDET GTA GIAI, dentro de la cual se ha trabajado en proyectos acreditados, se han formado tres doctores Nadal Mora (2005), Piechocki (2012), Martiarena (2018) (además, hay un doctorando en la actualidad) y ha permitido la vinculación con universidades de Europa como ser la Universidad Politécnica de Madrid. Por estas razones es que en la actualidad se está trabajando con la etapa de desarrollo y pruebas experimentales y por tal, fue necesario construir un túnel de viento vertical.

El mismo se encuentra desarrollado, calibrado y equipado con el objeto de medir la velocidad del aire, y todos los parámetros asociados a la rotación del pararrotor, con el objeto de simular la caída libre del mismo.

El presente trabajo describe el diseño del túnel con sus consideraciones, dimensiones y geometrías, la caracterización y sus rediseños, ensayos de prueba y las conclusiones.

\section{Diseño del tunel}

\subsection{Geometría y dimensiones}

El túnel se diseñó de manera modular (figura 1). El mismo consta de tres módulos de sección cuadrada. Dos módulos rectos y un módulo divergente (entre ambos) el cual oficia de sección de prueba. En la base y sujeto a una bancada de acero se ubica un soplador axial, encargado de propulsar una corriente de aire a la entrada del túnel. Se coloca en el primer módulo un enderezador de flujo.

A continuación, se describen los elementos constituyentes del túnel. 


\subsubsection{Módulo base}

Este módulo se ubica en la base del túnel y es por donde ingresa la corriente de aire. Se eligió una sección cuadrada, por cuestiones constructivas, con el fin de introducir el soplador dentro del módulo, condicionando de esta manera las dimensiones transversales del módulo (figura 2). El carenado del soplador tiene un radio exterior de $555 \mathrm{~mm}$.
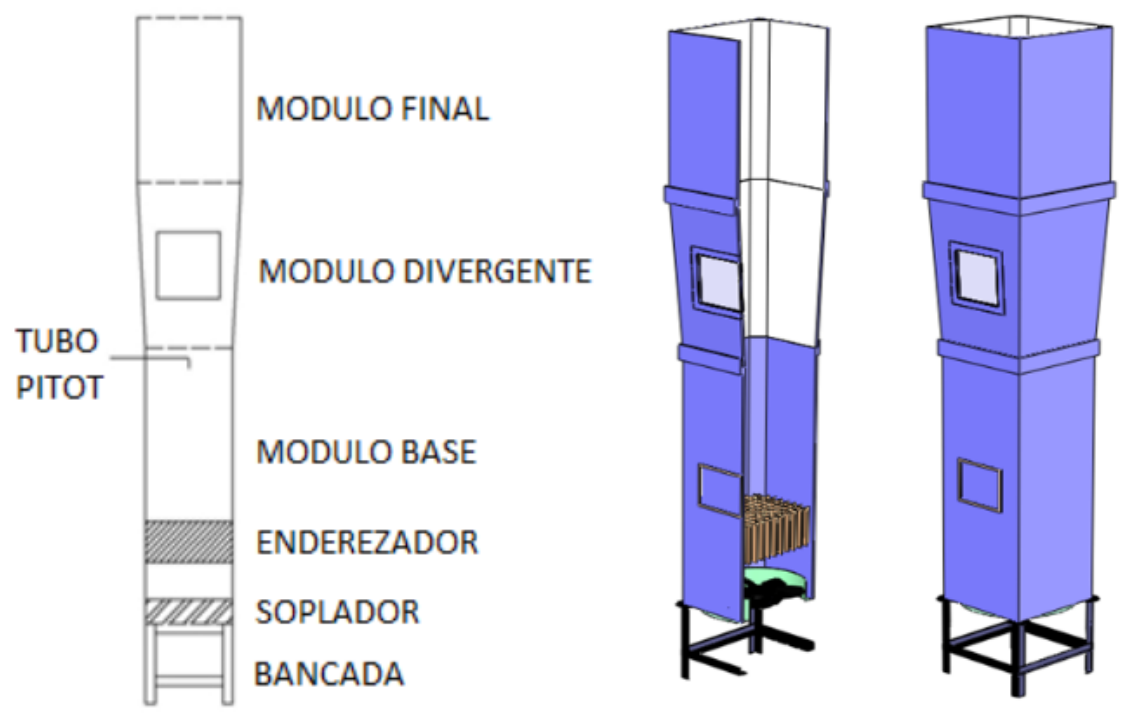

Figura 1: Esquema túnel de viento vertical.

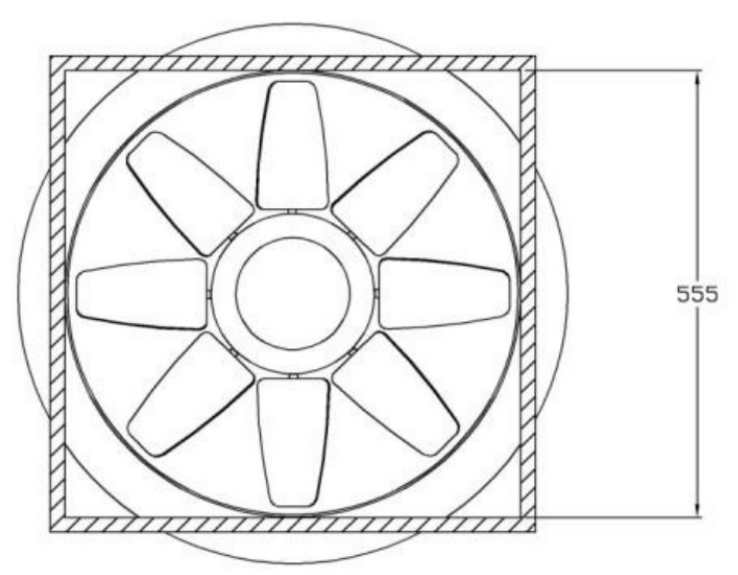

Figura 2: Carenado del soplador dentro del módulo base. Sección cuadrada del módulo base D1=555mm.

Por lo tanto, los lados de la sección cuadrada serán de D1: 555 mm.

El largo del módulo resultó producto de un análisis, entre la Norma IRAM 19004 (1996), la experiencia de la UIDET GTA GIAI en este tipo de dispositivos, la disponibilidad de los materiales de construcción (medidas de placas MDF), dimensiones de la sala de montaje del túnel y la funcionalidad del mismo. Resultando de esta manera, un largo de L1: $1670 \mathrm{~mm}$. Se diseñan también, en carácter de refuerzo, largueros en cada vértice de los módulos a $45^{\circ}$. De esta manera, también se eliminan los vértices rectos de la sección. 


\subsubsection{Módulo Divergente (sección de prueba)}

Este módulo se ubica por encima del módulo base y es donde se encuentra la sección de prueba, por lo tanto, es donde se busca un perfil homogéneo de velocidades. Se propone una divergencia en este módulo, es decir, un aumento gradual del área transversal, conforme nos desplazamos de manera ascendente, con el fin de generar un regulador de posición del objeto a ensayar en la sección de prueba.

La sección de prueba divergente funciona de la siguiente manera: ante una perturbación que incremente la velocidad, si la sección fuera constante el cuerpo ascendería, producto que ha aumentado la resistencia del mismo saliendo de la sección de prueba. Ahora bien, en el momento que el objeto ascendió, este se encuentra en una posición por encima de la sección de prueba, por lo tanto, el área transversal del módulo ha aumentado, por lo tanto, para mantener un caudal constante, la velocidad del túnel desciende (Figura 3). De esta manera, el cuerpo descenderá nuevamente a la posición de equilibrio. El regulador tendrá el efecto inverso, en caso de un descenso en la velocidad, el cuerpo descendería, se encontraría con una velocidad mayor y volvería a la posición de equilibrio.

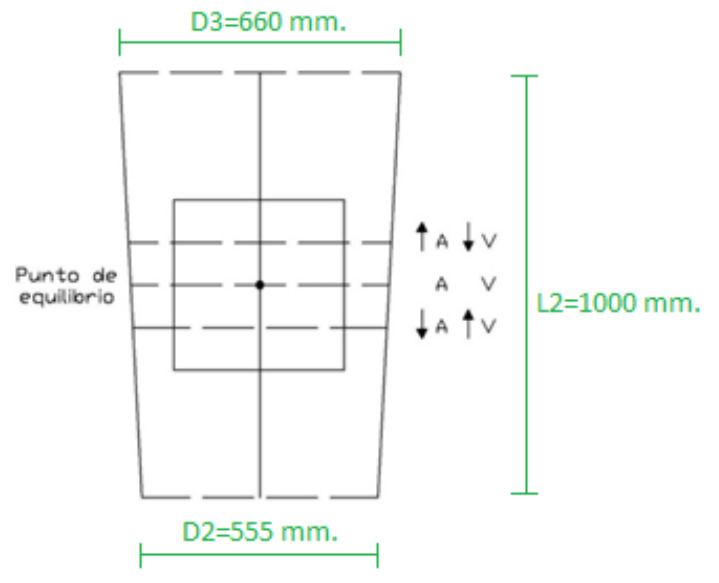

Figura 3: Módulo divergente con variación de área y velocidad.

Las dimensiones de entrada del módulo divergente serán las mismas que las del módulo base, ya que se busca una continuidad en las paredes interiores del túnel.

Por lo tanto, tenemos que D2: $555 \mathrm{~mm}$.

Se elige como longitud de este módulo, L2: $1000 \mathrm{~mm}$.

Para la divergencia del módulo, es decir, el ángulo de apertura o de incrementos de área de la sección del módulo, se consideró 3․ La Norma IRAM 19004 (1996) indica un máximo de 3.5. Utilizando un ángulo de $3^{\circ}$, y con la longitud (L2) del módulo, se define el área transversal de salida. Resultando el siguiente valor: D3: $660 \mathrm{~mm}$.

Con el fin de disponer de un espacio para armar el ensayo y la posterior visualización del mismo, a este módulo se le realizó una puerta-ventana frontal de 400 × 400 mm en la sección de prueba.

Así mismo, para optimizar la visualización del ensayo, a este módulo se lo pinto de blanco por dentro, de manera de tener un mejor contraste a la hora de evaluar el comportamiento del objeto a ensayar. 


\subsubsection{Módulo Final}

La función de este módulo es proporcionar un conducto luego de la sección de prueba: de esta manera la descarga de la corriente se ubica a una distancia que asegura no tener perturbaciones que afecten dicha sección. Nuevamente los lados de la sección transversal de este módulo, están condicionados por el área de salida del módulo divergente. Siendo este valor D3: $660 \mathrm{~mm}$ y la longitud propuesta del módulo, L3: $1000 \mathrm{~mm}$.

\subsubsection{Elementos adicionales del túnel}

\subsubsection{Enderezador de flujo}

Se incorporó un enderezador de flujos en el primer módulo del túnel, con el objeto de minimizar las componentes transversales de la corriente y uniformizar la distribución de velocidades. La Norma IRAM 19004 (1996) da las dimensiones y tolerancias del enderezador de flujo, de acuerdo al diámetro equivalente del túnel, $\mathrm{D}_{e}$ (figuras 4 y 5).
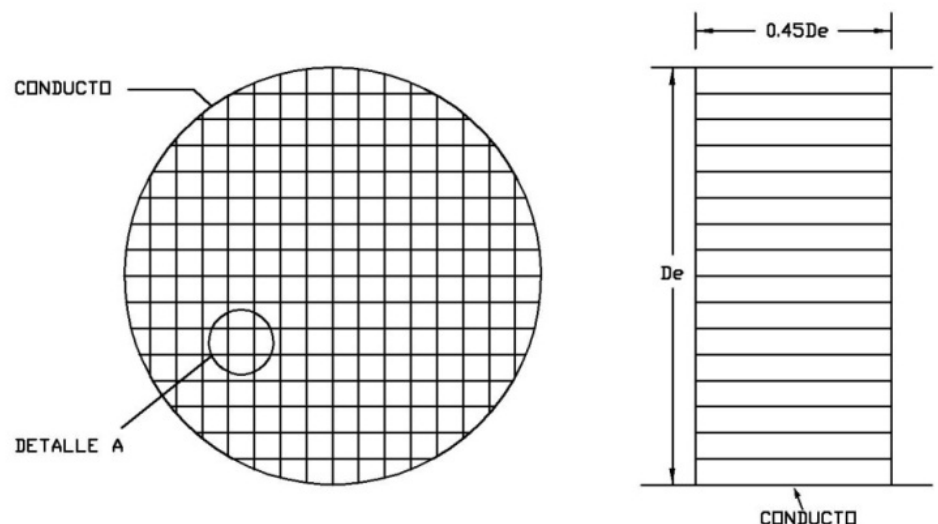

(cuadrado o circular de diámetro equivalente)

Figura 4: Dimensiones del enderezador de flujo - diámetro equivalente - Norma IRAM 19004 (1996)

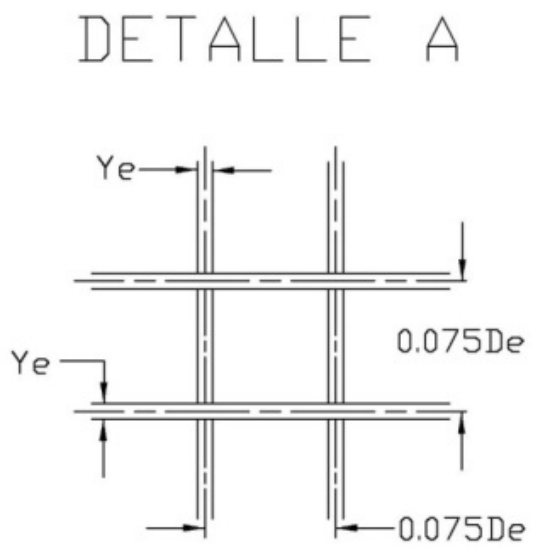

Figura 5: Detalle A dimensiones del enderezador del flujo - Norma IRAM 19004 (1996) 


\subsubsection{Soplador}

Como se mencionó en las consideraciones de diseño, la UIDET GTA GIAI de la Facultad de Ingeniería de la UNLP contaba con un soplador disponible para ser utilizado en el presente túnel.

El motor/soplador, es un electroventilador marca GATTI, modelo KRT 550/4 P. En la Tabla 1, se muestran los datos técnicos del motor.

Tabla 1: Datos técnicos Motor/soplador.

\begin{tabular}{|c|c|c|c|c|c|}
\hline \multicolumn{7}{|c|}{ Datos técnicos del Soplador } \\
\hline Modelo & $\varnothing$ Hélice $[\mathrm{mm}]$ & Motor $(50 \mathrm{~Hz})$ & Nivel sonoro [db A] & Peso [kg] \\
\cline { 3 - 5 } & & C.V. & R.P.M. & & \\
\hline KRT 550/4 P & 530 & 0,75 & 1350 & 76 & 16 \\
\hline
\end{tabular}

En la figura 6, se pueden ver las dimensiones del soplador.
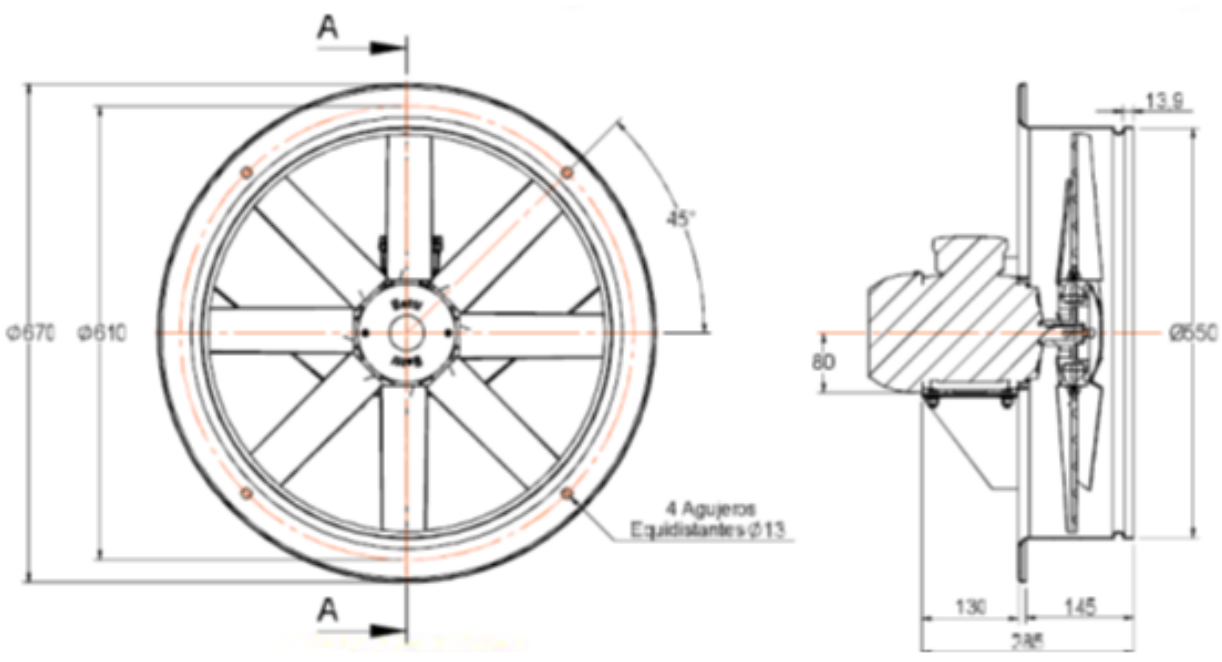

Figura 6: Dimensiones del soplador.

\subsection{Caracterización}

Con el fin de conocer el perfil de velocidades en la sección de prueba, se realizó una caracterización de dicha sección, para ello se tomaron medidas de la velocidad en diferentes puntos.

En referencia al equipo de laboratorio empleado:

- Tubo Pitot

- Regulador de voltaje

- Planilla para registro de datos

En referencia al instrumental utilizado: 
- Luz Estroboscópica Lutron. (Rango: De 100 a 10.000 rpm, Resolución 1 rpm)

- Micromanómetro diferencial R-Fuess. (Rango: De 0 a 260 mmCA, Resolución: 0,05 mmCA)

- Termómetro de bulbo seco - bulbo húmedo. (Alcance: bulbo húmedo de -25 a $30{ }^{\circ} \mathrm{C}$; bulbo seco de 0 a $100{ }^{\circ} \mathrm{C}$, Resolución: $1^{\circ} \mathrm{C}$ )

- Barómetro marca Druck. (Rango: 74500 a 115000 Pa, Resolución: 0,1 Pa)

Se realizaron 7 orificios equidistantes en la pared lateral derecha del túnel, a la altura de la sección de prueba, por estos orificios se introduce el tubo Pitot, el cual hará un barrido tomando 7 puntos nuevamente equidistantes entre sí, lo que da un total de 49 puntos de medida en la sección.

La configuración de dichos puntos se presenta en la figura 7.

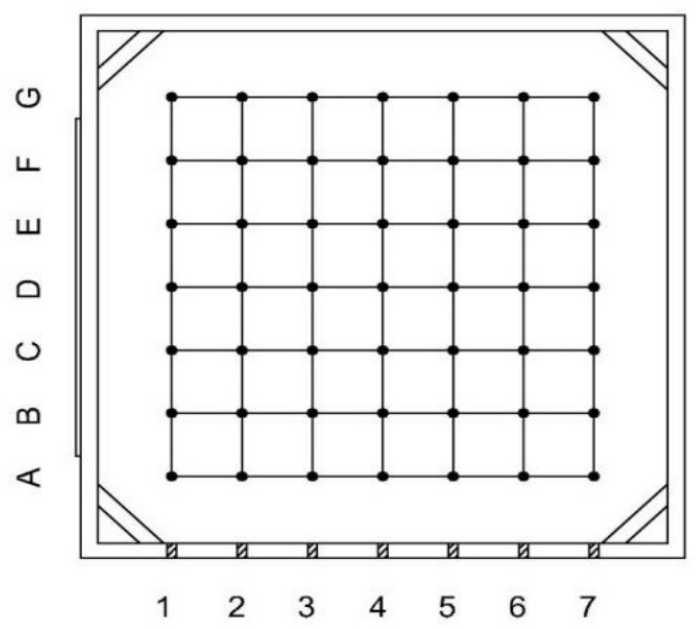

Figura 7: Puntos de caracterización.

Haciendo uso de la luz estroboscópica se midió la velocidad de giro que alcanzaba el soplador para 3 configuraciones. Las mismas son: $35 \mathrm{~Hz}$ y $50 \mathrm{~Hz}$ del regulador de voltaje y la última configuración con el soplador en conexión directa. Los valores se presentan en la Tabla 2.

Tabla 2: Velocidad de giro correspondiente a la configuración del soplador.

\begin{tabular}{|c|c|}
\hline Configuración del soplador & Velocidad de giro [rpm] \\
\hline $35 \mathrm{~Hz}$ & 1049 \\
\hline $50 \mathrm{~Hz}$ & 1230 \\
\hline Conexión directa (Trifásica) & 1440 \\
\hline
\end{tabular}

Se utilizó dicha variable del regulador de voltaje (frecuencia), para fijar la velocidad de giro deseada a la hora de realizar el ensayo. Previo a la toma de los valores de velocidad, con el objeto de conocer en una primera etapa el perfil de velocidades, se realizó un ensayo cualitativo para conocer como respondía el perfil de velocidades en la sección de prueba. A continuación, en la figura 8 se muestra un esquema del perfil de velocidades obtenido. 


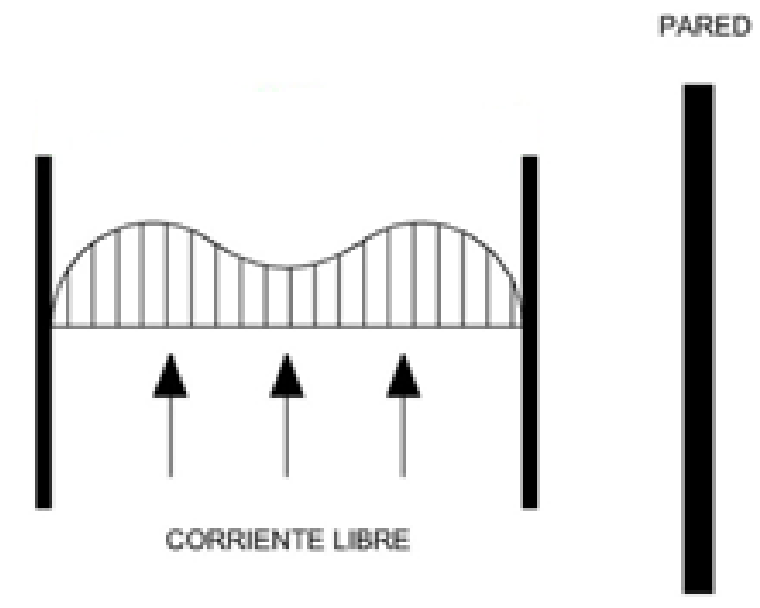

Figura 8: Esquema perfil de velocidades, primer ensayo cualitativo.

En el mismo, se aprecia una disminución de la velocidad en la región central. Del resultado de este primer ensayo se concluye lo siguiente: existe una clara relación entre el diámetro del eje del soplador con la región de menor velocidad en la sección de prueba (sector central); se concluye que esto ocurre en parte por las dimensiones del eje del soplador, un diámetro grande en relación a las palas; y por otro lado, en virtud de la distancia entre el soplador y la sección de prueba, ya que la misma no es suficiente para mitigar los efectos producidos por el eje. Esto último era de esperarse ya que, por cuestiones de diseño como se mencionó anteriormente, no se pudo respetar dicha distancia especificada por la Norma IRAM 19004 (1996).

\subsection{Rediseño}

Habiendo considerado fijas las variables tales como la longitud del túnel o la elección del soplador, se propuso como solución para corregir el perfil de velocidades, incorporar en el interior del túnel, a la salida del enderezador del flujo, una malla con un orificio en el centro copiando el diámetro del eje del soplador (figura 9); la misma está sujeta por un marco de madera que se fija a las paredes interiores del túnel (figura 10).

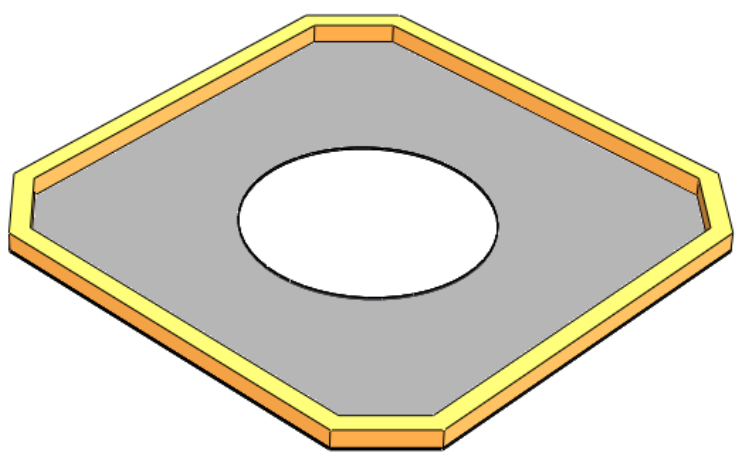

Figura 9: Modelado 3D de malla con orificio central. 


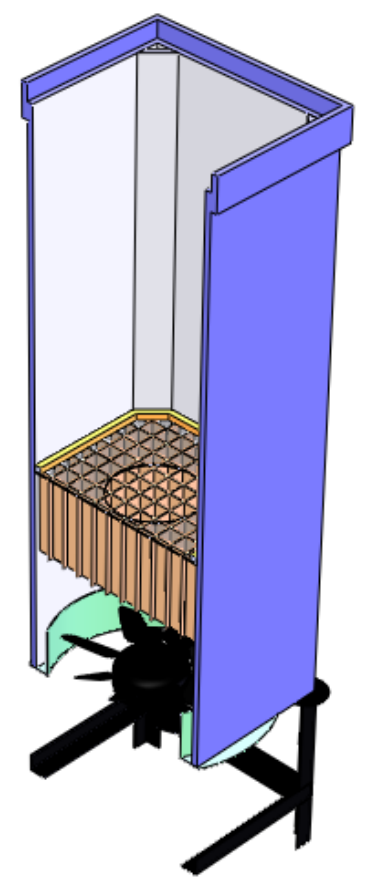

Figura 10: Modelado 3D del módulo base con el soplador, enderezador y malla.

De esta manera parte de la corriente que anteriormente ascendía libremente por los extremos, al encontrarse con el mallado, se direcciona al centro de la sección. La cantidad de láminas necesarias para lograr la proporción y el efecto deseado se determinó en función de los ensayos.

Nuevamente se realizó un ensayo cualitativo para ver como respondía el rediseño I. Fueron necesarias 3 láminas de telas metálicas para conseguir el efecto deseado en la sección de prueba.

Se logró mitigar la caída de velocidades en el centro de la sección de prueba, sin embargo, se aprecia una diferencia entre el centro del perfil de velocidades (área de prueba) y el centro geométrico de la sección (figura 11).

A continuación, se muestra un esquema del perfil de velocidades obtenido.

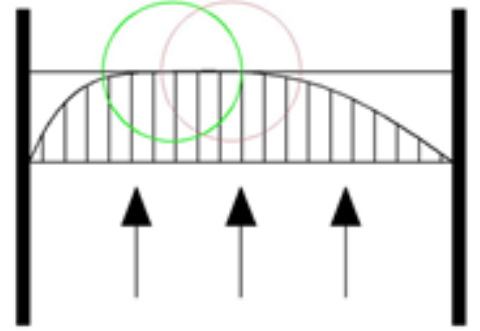

CORRIENTE LIBRE
PARED

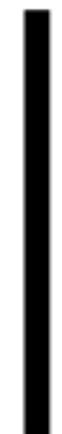

Figura 11: Esquema del perfil de velocidades, segundo ensayo. 
Se concluye que esta diferencia ocurre por un ingreso desigual de aire al soplador, producto de la cercanía del túnel con la pared de la sala de montaje (figura 12). Esta cercanía hace que en uno de los lados el ingreso de aire se vea acotado, mientras que en los lados restantes se puede considerar un ingreso libre.

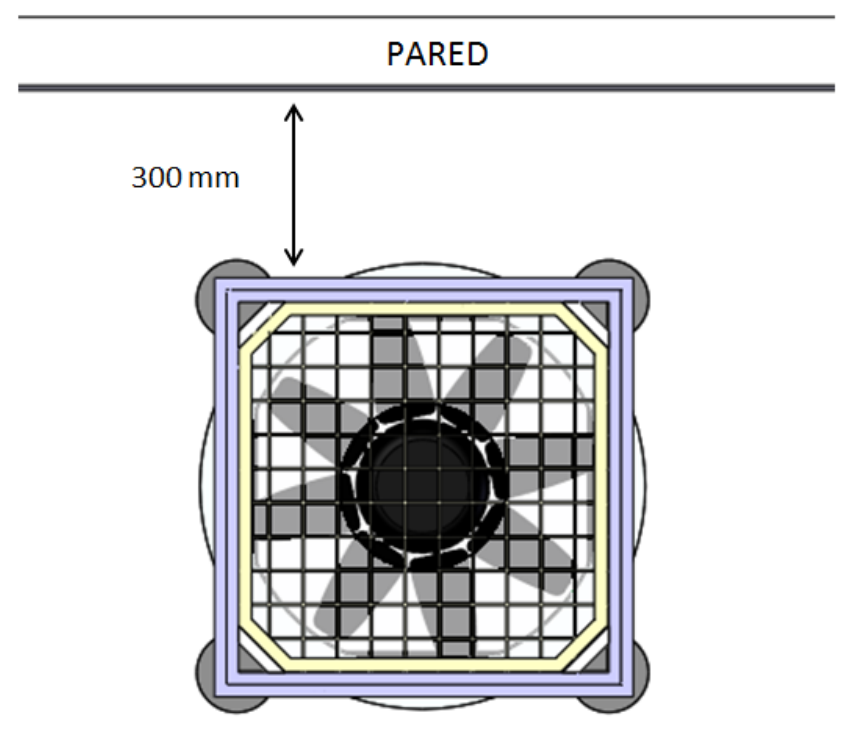

Figura 12: Vista superior modulo base, distancia a la pared.

A continuación, se propone un segundo rediseño como solución a dicho problema.

Ante la dificultad de desplazar el túnel hacia una nueva ubicación donde los muros no tengan efecto alguno sobre el ingreso de aire al soplador, se optó por construir un recinto alrededor del túnel, de manera tal, que exista la misma distancia entre el ingreso de aire al soplador y el muro, en cualquiera de sus ejes (figura 13).

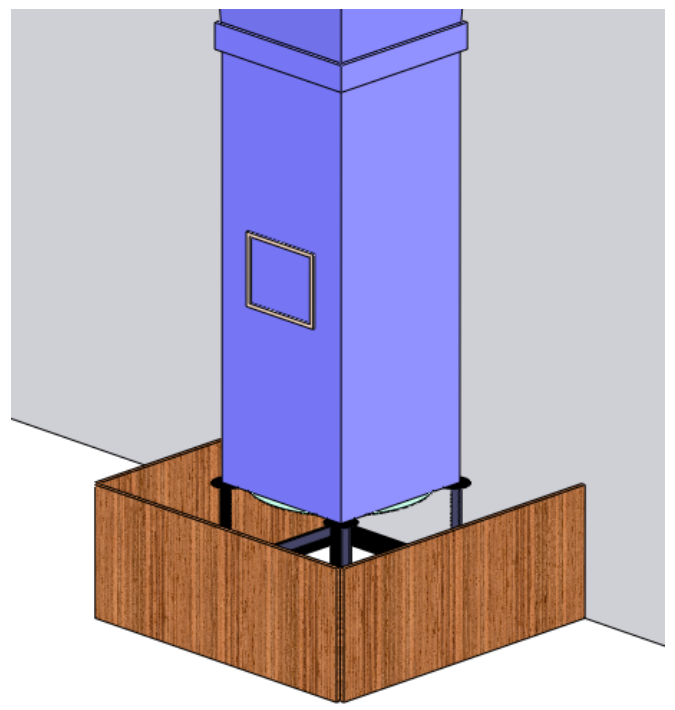

Figura 13: Modelado recinto equidistante. 


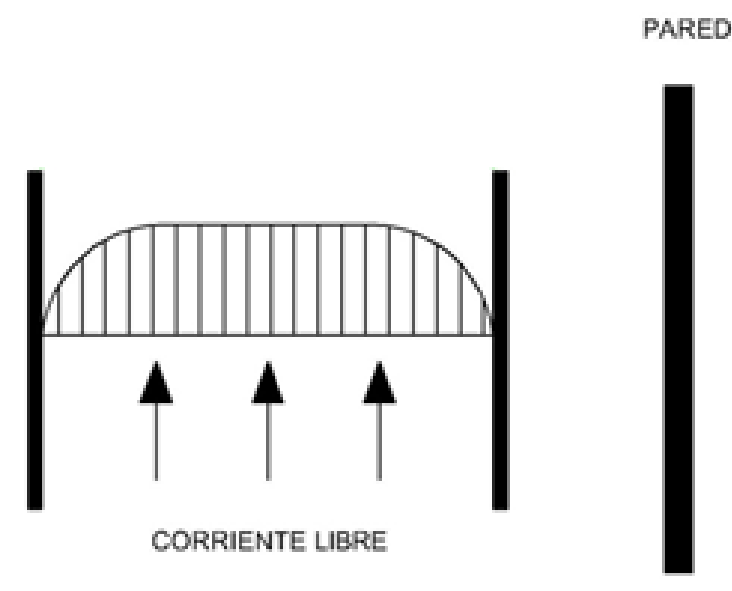

Figura 14: Esquema del perfil de velocidades, tercer ensayo cualitativo.

Se realizó nuevamente un ensayo cualitativo para ver la respuesta del perfil de velocidades ante el rediseño II, el perfil cualitativo obtenido se presenta en la figura 14.

Ante una respuesta favorable del perfil de velocidades, se procedió a la adquisición y registro de datos.

\subsection{Caracterización final}

Finalmente, conociendo el perfil de velocidades de manera cualitativa, se realizaron los ensayos de caracterización para tres velocidades de referencia $(4,23 \mathrm{~m} / \mathrm{s} ; 5,06 \mathrm{~m} / \mathrm{s} \mathrm{y} \mathrm{6,05} \mathrm{m/s).} \mathrm{A} \mathrm{continuación,} \mathrm{en} \mathrm{las}$ Figuras 15-17 se muestran los resultados de la caracterización punto a punto.
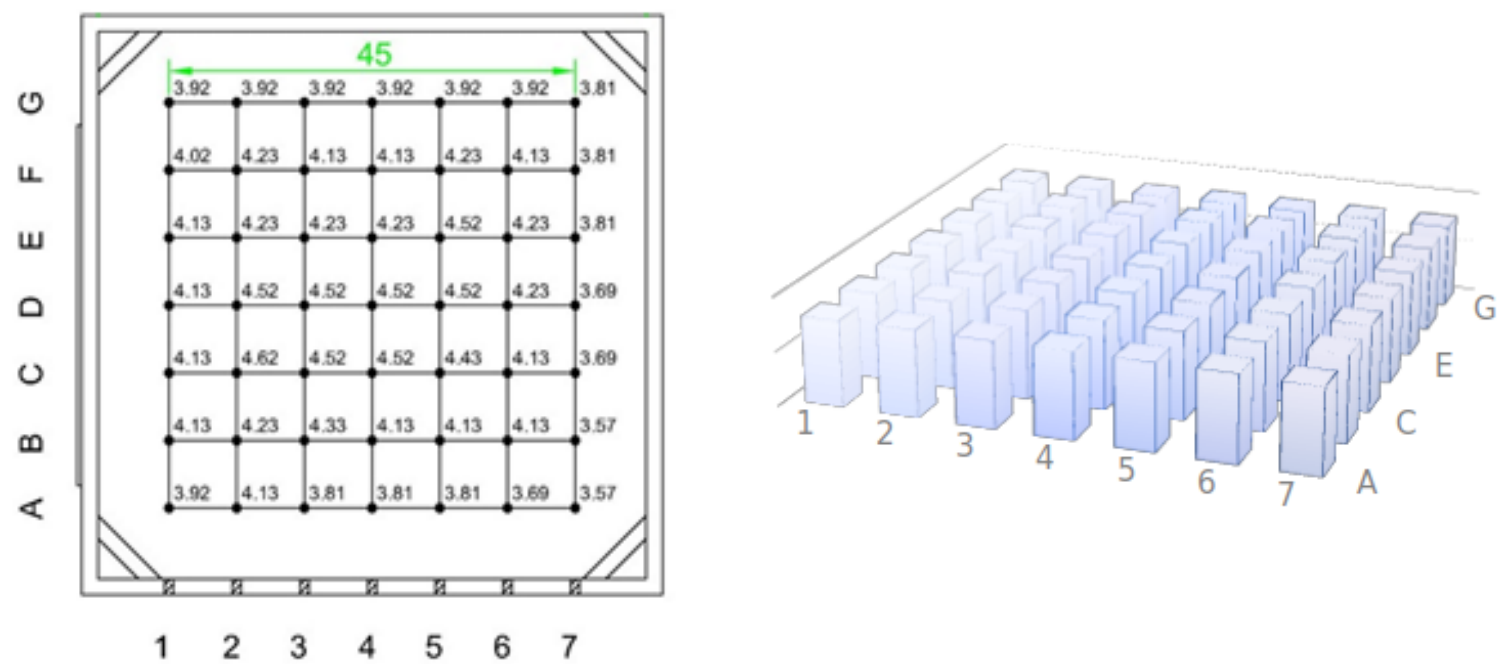

Figura 15: Valores de velocidad para una velocidad de referencia de 4,23 m/s. 

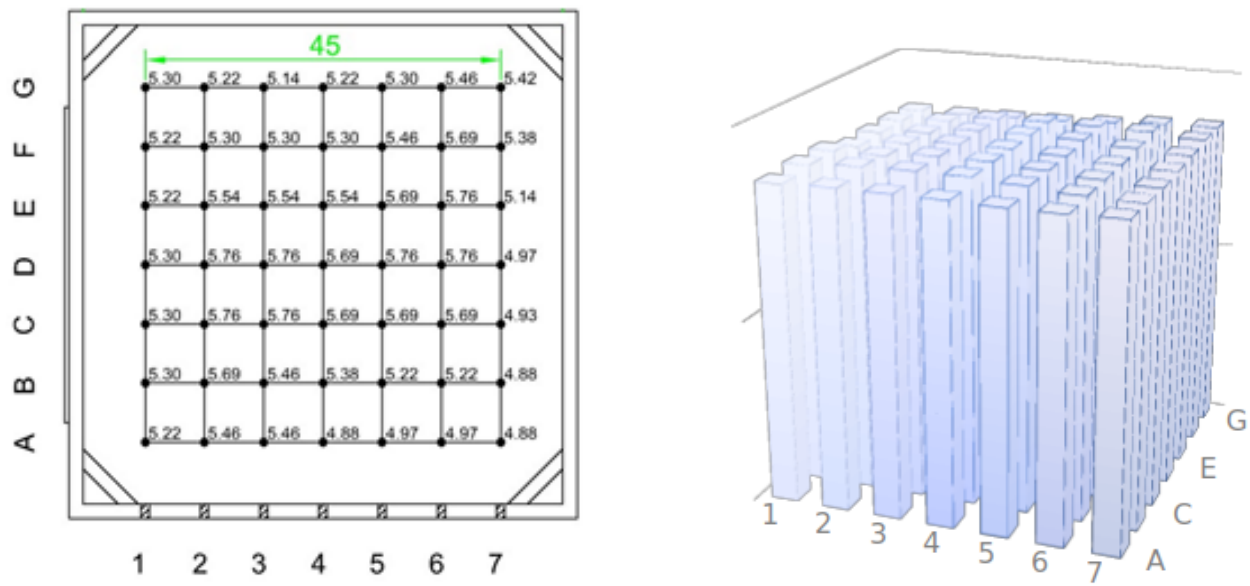

Figura 16: Valores de velocidad para una velocidad de referencia de 5,06 m/s.
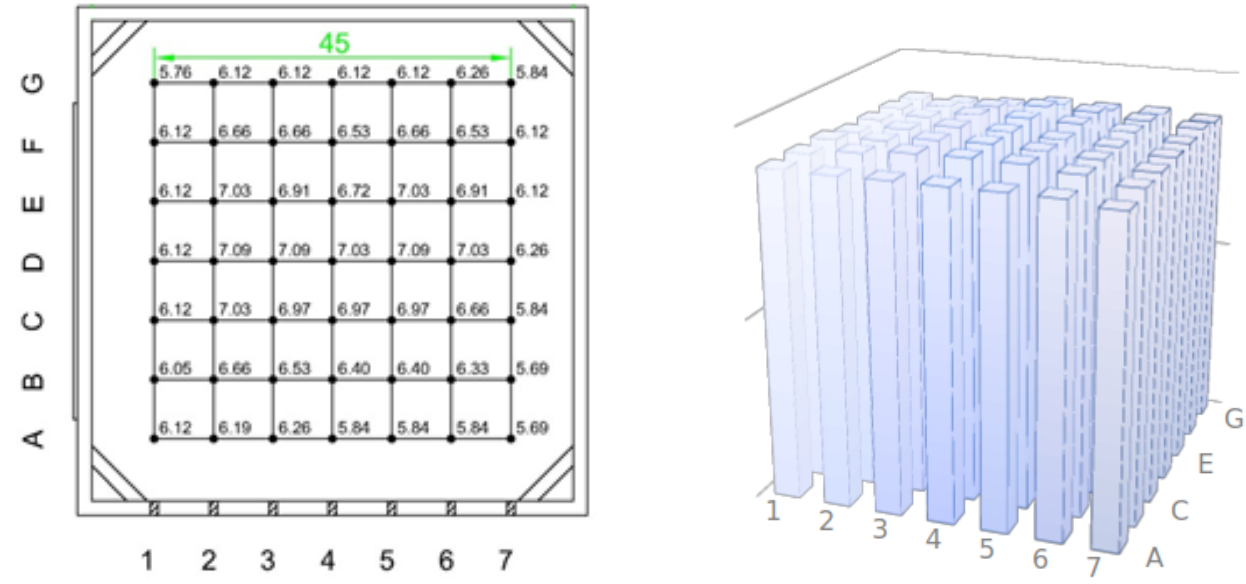

Figura 17: Valores de velocidad para una velocidad de referencia de $6,05 \mathrm{~m} / \mathrm{s}$.

En virtud de las mediciones de velocidad durante el ensayo de caracterización en este último rediseño (rediseño II), se concluye que se lograron mitigar los efectos producidos por el ingreso desigual de aire al soplador, por lo tanto el perfil de velocidades es simétrico respecto al eje longitudinal del túnel. Se divisa un área central de $30 \mathrm{~cm}$ de diámetro donde tenemos un perfil de velocidades apto para realizar los ensayos.

\section{Ensayo de prueba}

Para realizar ensayos de prueba del túnel y comprobar de esta manera el desempeño del mismo, se optó por ensayar un pararrotor cuya configuración (ángulo de paso de palas, radio de aspecto, dimensiones y momentos de inercia) correspondía a una condición cuyo comportamiento dinámico era conocido (figura 18). La construcción del prototipo se realizó mediante una impresora 3D. 


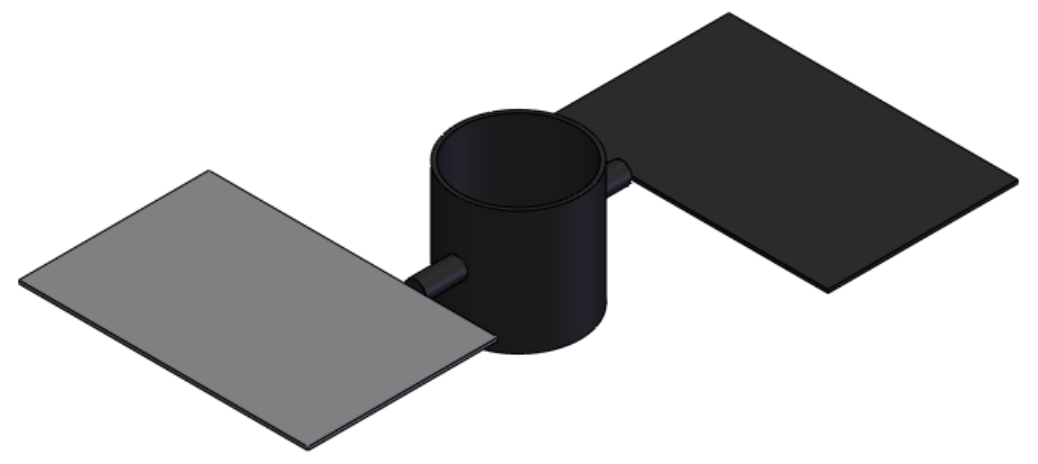

Figura 18: Modelado del prototipo.

Para realizar el ensayo de prueba, en primera instancia se colocaron dos varillas en el módulo de prueba, con el fin de sujetar el objeto a ensayar (ver Figura 19). En estas dos varillas se coloca un hilo, el hilo pasa por un orificio en el centro del objeto; de esta manera el cuerpo queda sujeto, pero libre de desplazarse verticalmente y de girar sobre su propio eje, y en menor medida (dependiendo de la tensión del hilo) puede desplazar lateralmente.
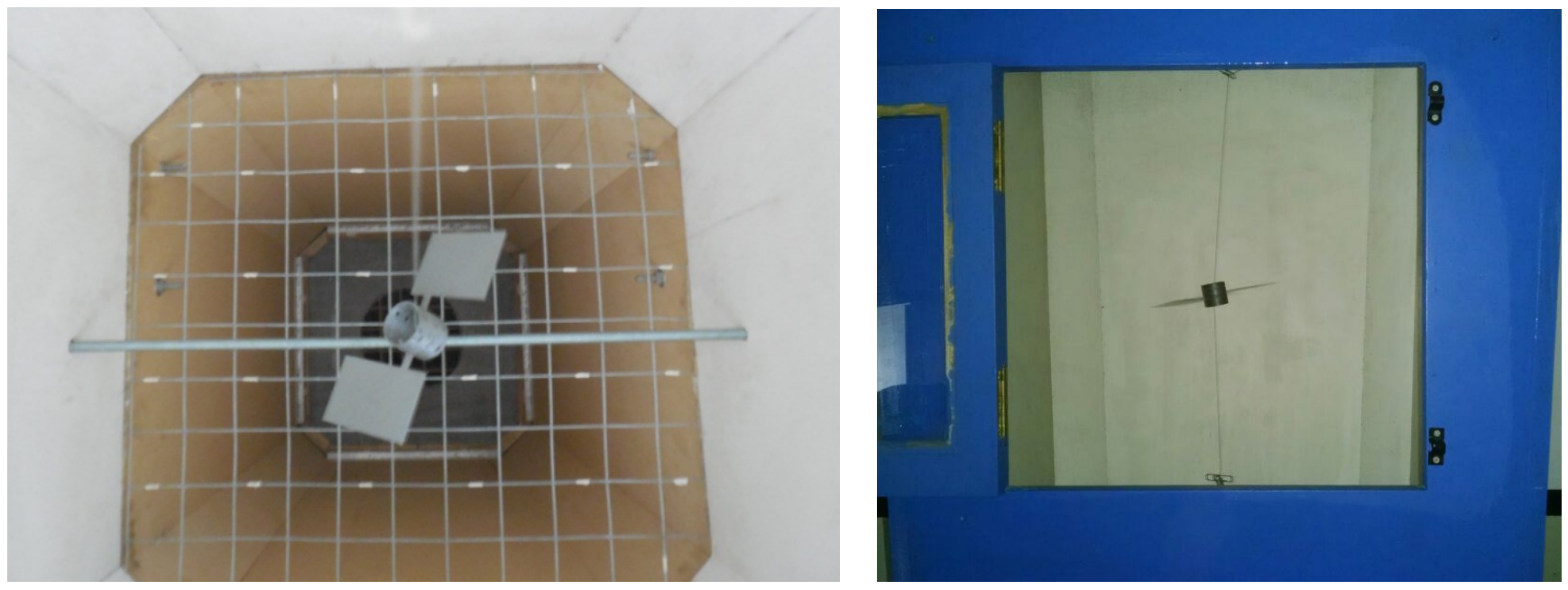

Figura 19: Modelado del prototipo.

Se observa una respuesta del prototipo acorde a lo esperado, ya que todos los parámetros medidos correspondieron con los modelos teóricos/analíticos. 


\section{Conclusiones}

Se concluye finalmente y en virtud de las conclusiones parciales obtenidas en cada uno de los ensayos realizados, que el túnel cuenta con una sección de prueba donde se tiene un perfil homogéneo de velocidades apto para realizar ensayos de objetos en caída libre.

La construcción de este túnel permitió la contrastación experimental de resultados obtenidos de manera analítica, que han sido publicados (Nadal Mora et al. (2005), Nadal Mora et al. (2006), Nadal Mora y Sanz Andrés (2006), Nadal Mora et al. (2007), Piechocki et al. (2014), Piechocki et al. (2016)) en diversas revistas científicas generando un desarrollo completo en el estudio de la temática.

\section{Referencias}

Martiarena, J. (2018). Estudio del desplazamiento lateral de un decelerador de alas rotatorias de pequeño alargamiento a través de las variaciones cíclicas del paso de las palas. Tesis doctoral, Facultad de Ingeniería.

Nadal Mora, V. (2005). Comportamiento Aerodinámico de Sondas Atmosféricas en Entornos Aeroportuarios. Tesis doctoral, Universidad Politécnica de Madrid, Madrid.

Nadal Mora, V., Piechocki, J., y Sanz Andres, A. (2007). Experimental research on a vertically falling rotating wing decelerator model. AIAA Paper.

Nadal Mora, V. y Sanz Andrés, A. (2006). Stability analysis of a free falling pararotor. Journal of Aircraft, $43: 980-986$.

Nadal Mora, V., Sanz Andrés, A., y Cuerva, A. (2005). Experimental investigation of an autorotatingwing aerodynamic decelerator system. AIAA Paper 2005-1635.

Nadal Mora, V., Sanz Andrés, A., y Cuerva, A. (2006). Model of the aerodynamic behaviour of a pararotor. Journal of Aircraft, 43:1893 - 1903.

Norma IRAM 19004 (1996).

Piechocki, J. (2012). Estudio de la Dinámica del Vuelo de un Decelerador Aerodinámico Basado en el Concepto de Pararrotor. Tesis doctoral, Facultad de ingeniería.

Piechocki, J., Nadal Mora, V., y Sanz Andrés, A. (2014). Pararotor dynamics: Center of mass displacement from the blade plane-analytical approach. Journal of Aircraft, 51:651-660.

Piechocki, J., Nadal Mora, V., y Sanz Andrés, A. (2016). Numerical simulation of pararotor dynamics: Effect of mass displacement from blade plane. Aerosp. Sci. Technol, 55:400 - 408. 